\title{
Diseño de unidades STEM integradas: una propuesta para responder a los desafíos del aula multigrado
}

\author{
Design of Integrated STEM Units: A Proposal to Respond to the Challenges of the \\ Multigrade Classroom
}

\section{Projeto de unidades STEM integradas: uma proposta para responder aos desafios da sala de aula multisseria}

\author{
Angela Castro-Inostroza ${ }^{1}$ \\ Rodrigo Jiménez-Villarroel ${ }^{2}$ \\ Jhonny Medina-Paredes 3 (D)
}

Recibido: abril de 2021

Aceptado: agosto de 2021

Para citar este artículo: Castro-Inostroza, A., Jiménez-Villarroel, R., y Medina-Paredes, J. (2021). Diseño de unidades STEM integradas: una propuesta para responder a los desafíos del aula multigrado. Revista Científica, 42(3), 339-352. https://doi.org/10.14483/23448350.17900

\section{Resumen}

La enseñanza multigrado tiene el desafío de garantizar oportunidades de acceso a experiencias de aprendizaje auténticas y relevantes, tal como aquellas a las que pueden optar los alumnos en contextos tradicionales de escolaridad. En este artículo de reflexión argumentamos que una forma de hacer frente a este y otros desafíos de la enseñanza en contextos multigrado es implementar la educación STEM, apoyando y alentando a los docentes a diseñar unidades curriculares STEM integradas. Comenzamos este artículo compartiendo algunas consideraciones sobre los principales desafíos de la educación multigrado y el potencial que tiene la educación STEM integrada para abordarlos. A continuación, analizamos el propósito de diversas estrategias de enseñanza en contextos multigrado reportadas en la literatura y exponemos los principales desafíos asociados al diseño de unidades STEM integradas, identificando aspectos clave que se deben considerar para desarrollar este tipo de propuestas en tales contextos. Finalmente, sobre la base de los aspectos identificados, ofrecemos una alternativa para acercar la educación STEM al aula multigrado, proponiendo un marco que oriente el diseño de unidades STEM integradas en estos contextos.

Palabras clave: educación multigrado; enfoque interdisciplinario; enseñanza primaria; estrategias educativas; plan de estudios integrado.

1. Universidad Austral de Chile, Puerto Montt, Chile, agela.castro@uach.cl

2. Universidad Austral de Chile, Puerto Montt, Chile, rodrigo.jimenez@uach.cl

3. Universidad Austral de Chile, Puerto Montt, Chile, jhonnymedina@uach.cl 


\begin{abstract}
Multigrade teaching is challenged to ensure opportunities for access to authentic and relevant learning experiences, such as those available to students in traditional schooling contexts. In this reflection article, we argue that one way to address this and other challenges of teaching in multigrade contexts is to implement STEM education by supporting and encouraging teachers to design integrated STEM curricular units. We begin this article by sharing some considerations about the main challenges of multigrade education and the potential of integrated STEM education to address them. Next, we analyze the purpose of various teaching strategies in multigrade contexts reported in the literature, and we expose the main challenges associated with the design of integrated STEM units, identifying key aspects that must be considered to develop this type of proposals in such contexts. Finally, based on the identified aspects, we offer an alternative to bring STEM education closer to the multigrade classroom by proposing a framework to guide the design of integrated STEM units in these contexts.
\end{abstract}

Keywords: educational strategies; integrated curriculum; interdisciplinary approach; multigrade education; primary education.

\section{Resumo}

O ensino multisseriado enfrenta o desafio de garantir oportunidades de acesso a experiências de aprendizagem autênticas e relevantes, como aquelas que os alunos podem escolher em contextos tradicionais de escolarização. Neste artigo reflexivo, defendemos que uma forma de enfrentar este e outros desafios do ensino em contextos multisserários é implementar a educação STEM, apoiando e incentivando os professores a conceber unidades curriculares STEM integradas. Começamos este artigo compartilhando algumas reflexões sobre os principais desafios da educação multisseriada e o potencial que a educação STEM integrada tem para enfrentá-los. A seguir, analisamos o propósito de várias estratégias de ensino em contextos multisseriados relatados na literatura e expomos os principais desafios associados à conceção de unidades STEM integradas, identificando os principais aspectos que devem ser considerados para desenvolver este tipo de propostas em tais contextos. Por fim, com base nos aspectos identificados, oferecemos uma alternativa para aproximar o ensino CTEM da turma multisseriada, propondo um quadro que norteie a concepção de unidades CTEM integradas nestes contextos.

Palavras-chaves: abordagem interdisciplinar; currículo integrado; educação multisseriada; ensino fundamental; estratégias educacionais.

\section{Introducción}

La enseñanza en contextos multigrado es común, tanto en países desarrollados como en aquellos en vías de desarrollo (Hyry-Beihammer y Hascher, 2015) y su implementación obedece a la necesidad de dar respuesta a distintas problemáticas, entre las que destacan áreas rurales o zonas de influencia urbana donde la población es escasa y las distancias son demasiado grandes para que los estudiantes viajen todos los días a un centro urbano (Taole, 2017; Vera-Bachman y Salvo, 2016). Para comunidades pequeñas, que a menudo se ubican en regiones remotas y aisladas, las escuelas multigrado se transforman en una alternativa viable para garantizar el derecho a la educación de los niños que viven en tales contextos (UNESCO, 2015).

A pesar de no ser una tarea fácil, la enseñanza multigrado tiene la obligación de garantizar a los niños que allí se educan experiencias de aprendizaje que los preparen para un futuro más prometedor (UNESCO, 2015). Estas experiencias, de la mano con el currículum nacional, deben permitirles la adquisición de conocimientos y el desarollo de habilidades que les permitan participar y discernir respecto de problemáticas locales y globales del siglo XXI, las cuales tienen un carácter notoriamente interdisciplinar (Castro et al., 2020).

La enseñanza multigrado es compleja y requiere un modelo de actuación específico (Bustos, 2014) para garantizar su calidad. Paradójicamente, muchos docentes carecen de una formación que los prepare para este tipo de enseñanza (Taole, 
2017). Además, las investigaciones sobre los procesos de enseñanza-aprendizaje en tales contextos ha sido escasa (Hyry-Beihammer y Hascher, 2015; Shareefa, 2021). Estudios disponibles (p. e. Abós Olivares y Bustos-Jiménez, 2015; Abós Olivares y Boix, 2017; Ribadeneira, 2020) evidencian el desafío y las dificultades que supone para los docentes la adaptación del currículo para la enseñanza de varios grados a la vez sin homogeneizar a los estudiantes, así como la escasez de materiales para apoyar esta tarea.

Una forma de hacer frente al reto que experimentan los profesores multigrado en el desarrollo de experiencias de aprendizaje auténticas y relevantes para los escolares —que promuevan el aprendizaje activo, la circulación y apropiación de saberes, el desarrollo de habilidades del siglo XXI y el abordaje de problemáticas locales y globales con un carácter interdisciplinar, respetando la heterogeneidad propia de estos contextos- es apoyar y alentar a los docentes a diseñar unidades curriculares STEM integradas. La educación STEM integrada se plantea como un enfoque educativo multidisciplinario que remueve las barreras tradicionales de la ciencia, la tecnología, la ingeniería y las matemáticas, y las integra al mundo real con experiencias rigurosas y relevantes que promueven oportunidades para que los estudiantes fomenten su conciencia sobre cómo se combinan los temas o dominios aparentemente no relacionados al abordar problemáticas reales (Nadelson y Seifert, 2019). Aunque en sus orígenes la forma de concebir la educación STEM estaba basada en imperativos económicos y vocacionales (Blackley y Howell, 2015), con el trascurso de los años esta visión ha avanzado a una concepción formativa, orientada a preparar a los ciudadanos del siglo XXI para enfrentar problemas multidisciplinarios que se dan en contextos reales, alfabetizándolos en estos campos y alentándolos a seguir carreras STEM (Bosch et al., 2011; Breiner et al., 2012; Guzey et al., 2016; Nadelson y Seifert, 2017; Moore et al.; 2015).

El aprendizaje activo e interdisciplinario en STEM tiene el potencial para preparar a los ciudadanos con las herramientas necesarias para vivir y contribuir en la sociedad (Castro et al., 2020; Ortiz-Revilla et al., 2020), así como para hacer el currículum más útil, relevante y motivador para los estudiantes (Nesmith y Cooper, 2019; Rennie et al., 2018). Además permite abordar variados temas con distintos grados de profundidad y ofrece diferentes formas de expresar lo que se ha aprendido, junto con distintas maneras de desarrollar los conocimientos y las habilidades involucradas (Clayton et al., 2010), aspectos clave para abordar la heterogeneidad en contextos multigrado (Shareefa, 2021).

Se han desarrollado numerosos estudios que proporcionan evidencia del valor de la educación STEM integrada en contextos formales de enseñanza tradicional (p. e. English y King, 2015 y 2019; Koul et al., 2018; Lehrer y Schauble, 2012; McDonald, 2016; Ng y Chan, 2019; Struyf et al., 2019; Tati et al., 2017, entre otros), destacando el valor de este tipo de experiencias en la formación los ciudadanos del siglo XXI, pues permite la alfabetización en estos campos y la promoción del desarrollo de las denominadas habilidades del siglo XXI (Castro et al., 2020). Sin embargo, a pesar del potencial de la educación STEM para hacer frente a muchos de los desafíos señalados de la enseñanza multigrado, el cómo acercarla a este contexto es algo que no ha sido explorado.

\section{Propósito de las estrategias de enseñanza en contextos multigrado}

Las aulas multigrado son un espacio de encuentro y convivencia para niños de distintas edades y grados de escolaridad. Esta realidad hace de la enseñanza en estos contextos una tarea compleja, que requiere el uso de estrategias pedagógicas específicas que contemplen la organización del espacio y el tiempo, así como la selección de los recursos curriculares más idóneos (Peirano et al., 2015).

En función de la conformación de los grupos y la organización de los temas, se han propuesto algunas estrategias que pueden ser útiles para 
abordar los procesos de enseñanza y aprendizaje en estos contextos. Así, por ejemplo, autores como Hyry-Beihammer y Hascher (2015) han reportado prácticas utilizadas por los docentes orientadas a disminuir la heterogeneidad de los estudiantes, con el objetivo de reducir el número de grupos con el que se debe trabajar en el aula y, así, facilitar la gestión de estas. Estas corresponden al currículum paralelo, es decir, todos los estudiantes comparten los mismos temas o materias pero estudian el programa de estudios de su grado; la rotación del plan de estudios, en donde toda la clase estudia el plan de estudios de un grado durante un año y en el próximo año escolar sigue el programa de estudios para el otro grado; la enseñanza de toda la clase, en la que todos los los alumnos estudian el mismo tema, al mismo tiempo, usando el mismo material; y el escalonamiento de materia, en que los diferentes grados en el aula trabajan en diferentes materias, con cada grado enseñado por turno, mientras que los otros estudiantes trabajan independientemente. En esta misma línea, UNESCO (2015) propone la enseñanza de todos los grados a la vez (misma metodología, contenido y resultados esperados) y enseñanza de un grado, mientras que los otros trabajan independientemente (breve instrucción directa con cada nivel y trabajo autónomo alternado).

También se ha reportado y propuesto un segundo tipo de prácticas orientadas a la instrucción diferenciada con el objetivo de respetar la heterogeneidad de los estudiantes. Entre estas, el método del currículo espiral, a través del cual los estudiantes comparten los mismos temas o materias pero el contenido está organizado en función de diferentes etapas, niveles o necesidades de los alumnos; la elaboración de planes de trabajo personales, que tiene sus raíces en las teorías educativas de la pedagogía Montessori y está orientada a promover la autonomía a través de la elección de sus tareas, materiales, horarios, lugares y formas de aprender; y la tutoría entre pares, ya sea de manera espontánea o incentivada por los maestros para promover la circulación de saberes (Hyry-Beihammer y Hascher 2015). De manera similar, UNESCO (2015) propone la enseñanza de un contenido para todos con diferentes niveles de dificultad (agrupamiento por grado, edad y habilidad para la enseñanza de un mismo tema en distinto nivel), actividades para grupos no enseñados (grados superiores investigan y recopilan información autónomamente); y estrategias de enseñanza entre pares, edades y grados cruzados (tutoría basada en el emparejamiento por edad).

Estas estrategias han sido recogidas por algunos países e incorporadas en las orientaciones ministeriales que se dan para las prácticas pedagógicas en contextos multigrado, como es el caso de Chile (Mineduc, 2017a). En este contexto se ha señalado la libertad que tienen los docentes para elegir el momento y las estrategias a utilizar, destacando el potencial que tiene para el aprendizaje planificar cuidadosamente cómo y cuándo utilizarlas (Mineduc, 2017b; UNESCO, 2015). No obstante, la evidencia muestra que no siempre se hace una combinación de estas, predominando el uso de estrategias más orientadas a homogenizar a los alumnos, sin promover la circulación de saberes (p. e. Boix y Bustos, 2014; Hyry-Beihammer y Hascher, 2015; Taole, 2020).

La investigación en el área evidencia que la enseñanza multigrado requiere del uso de estrategias activas de enseñanza, con un enfoque global e integral del currículum y una mezcla alternativa de espacios compartidos y espacios individuales que permitan el aprendizaje por contagio, el aprendizaje cooperativo, la enseñanza recíproca y el desarrollo de la autonomía (p. e. Abós Olivares y Bustos-Jiménez, 2015). Se destaca, así, el éxito de prácticas basadas en metodologías activas y participativas, acompañamiento pedagógico, métodos cooperativos o colaborativos como el aprendizaje basado en proyectos y prácticas que toman en cuenta de manera explícita la heterogeneidad de los estudiantes, como los modelos de aprendizaje en espiral (p. e. Abós Olivares y Bustos-Jiménez, 2015; Abós Olivares y Boix, 2017; Boix y Bustos, 
2014; Hyry-Beihammer y Hascher, 2015; Ribadeneira, 2020; entre otros).

Recientemente, han comenzado a investigarse los efectos de la instrucción diferenciada o diferenciación como estrategia para hacer frente a la heterogeneidad del aula multigrado (p. e. Shareefa, 2021; Taole, 2020). Esta implica la mediación del currículo para la enseñanza, el nivel del contenido y los materiales para la planificación y ejecución de actividades diferenciadas que den respuesta a las distintas necesidades de aprendizaje en una clase en que las edades pueden variar hasta en siete años (Taole, 2017). De acuerdo con Tomlinson (2014), la diferenciación puede describirse como una práctica en el aula que busca un equilibrio en el énfasis entre los estudiantes individualmente y el contenido del curso. Lo anterior se puede plantear en términos de una diferenciación del contenido, los procesos y los productos, en función de las capacidades, los intereses y los perfiles de aprendizaje de los estudiantes. La diferenciación de contenido hace referencia a conocimientos, conceptos y habilidades que los estudiantes deben aprender y desarrollar, y a la forma en que ellos acceden a estos (por ejemplo, a través de lecturas, investigación individual, comprensión auditiva, etc.). La diferenciación de proceso tiene relación con el cómo se logra que los estudiantes comprendan o den sentido al contenido (por ejemplo, a través del aprendizaje basado en proyectos, problemas, fenómenos, etc.). La diferenciación de producto hace referencia a las distintas formas mediante las cuales los estudiantes muestran lo que han aprendido y son capaces de hacer después de un tiempo determinado.

En contextos multigrado, la diferenciación se ha definido como el conjunto de experiencias de aprendizaje que los profesores brindan a sus alumnos después de evaluar detenidamente sus necesidades y habilidades con el objetivo de maximizar sus aprendizajes, independientemente de sus capacidades o grados designados (Shareefa, 2021). Lo anterior se considera un aspecto crucial dentro de la base de conocimientos profesionales que los profesores multigrado necesitan para la enseñanza (Taole, 2017) y resulta todo un desafío para ellos (Taole, 2017 y Taole, 2020). Posiblemente esto se deba a que, a pesar de que los programas de formación docente realzan la diferenciación, esta no se ha centrado en el contexto de las clases multigrado, ni en cómo adaptar el plan de estudio de enseñanza básica para ello, provocando que los docentes multigrado puedan tener problemas para utilizarla y no disponer de materiales adecuados para implementarla (Hyry-Beihammer y Hascher, 2015).

La instrucción diferenciada parece ser una estrategia prometedora para abordar la heterogeneidad del aula multigrado. No obstante, se requiere más investigación en el área que permita describir una forma de adaptar los planes de estudio considerando esta estrategia como base, así como determinar sus efectos en el aprendizaje de los alumnos en contextos educativos multigrado (Smit y Humpert, 2012).

\section{Los desafíos del diseño de lecciones STEM integradas}

La educación STEM integrada se plantea como un enfoque educativo que involucra a los estudiantes en contextos auténticos de aprendizaje, espacio que fomenta su comprensión sobre temas y problemas del mundo real —que tienen un carácter interdisciplinar-, preparándolos con los conocimientos y habilidades necesarias para el siglo XXI que les permitan posicionarse en temas asociados a estas áreas (Bennet y Ruchti, 2014; English y King, 2019, Guzey et al., 2016; Sias et al., 2017). Se conceptualiza como un enfoque centrado en el aprendizaje activo de los estudiantes, basado en problemas o proyectos que requieren para su solución, el aprendizaje y la aplicación del conocimiento de múltiples disciplinas STEM e involucra el uso de habilidades del siglo XXI, como por ejemplo, la colaboración, la creatividad, el pensamiento crítico y la comunicación (Nadelson y Seifert, 2017 y 2019). 
Se han documentado experiencias innovadoras de aprendizaje interdisciplinario en STEM, por ejemplo, la construcción de puentes de papel para carga (English y King; 2019), el diseño de cohetes (English y King, 2015), el diseño de rampas para acceso de sillas de ruedas (Tytler et al., 2019), el diseño y modelado en 3D de un vecindario (Shealer y Shealer, 2014), entre otras. Tales experiencias sugieren que integrar, al menos, parte del plan de estudios STEM genera un currículo relevante y motivador (Rennie et al., 2018). Sin embargo, existen desafíos asociados a la interdisciplinariedad en STEM que complejizan el diseño de unidades STEM: por ejemplo, consensuar una definición para la integración y mantener la integridad epistémica de las áreas integradas (English, 2016; Honey et al., 2014; Tytler et al., 2019).

Hay diferentes perspectivas de qué significa un plan de estudios integrado y se han establecido diversas categorías de integración (p. e. English, 2016; Gresnigt et al., 2014; Rennie et al., 2018). La mayoría de ellas comienza con planes de estudio de disciplinas que se enseñan separadamente para luego describir grados de superposición de estas (Rennie et al., 2018). Así, por ejemplo, se incluyen enfoques disciplinarios, fragmentados o sincronizados (conceptos y habilidades se aprenden por separado en cada disciplina, estableciendo una conexión explícita entre estas), interdisciplinarios (conceptos y habilidades entre disciplinas se desarrollan estrechamente vinculados) y transdisciplinarios (conocimientos y habilidades entre disciplinas se aplican a problemas y proyectos reales) (English, 2016; Rennie et al., 2018). No obstante, es necesario atender la naturaleza temporal de las interacciones entre las áreas integradas y la existencia de límites difusos entre estas (English, 2016; Honey et al., 2014; Ortiz-Revilla et al., 2020).

Autores como Tytler et al. (2019) señalan que la descripción - como áreas que se cruzan o regiones superpuestas - de las interacciones entre las disciplinas STEM integradas es útil, por ejemplo, para determinar qué parte del plan de estudios se podría integrar, pero insuficiente para determinar cómo los conceptos asociados interactúan en el tiempo. Estos autores señalan que el entrelazamiento de los conocimientos disciplinarios se produce en diferentes escalas de tiempo, tanto para los alumnos (al resolver un problema), como para los profesores (al introducir ideas y prácticas disciplinarias).

Otra problemática asociada a la interdisciplinariedad en STEM es la pérdida de integridad epistémica de las áreas que se integran (English, 2016; Tytler et al., 2019). Las unidades integradas no siempre median una construcción profunda del conocimiento en las áreas involucradas (Chalmers et al., 2017), destacándose, por ejemplo, que no todas las matemáticas pueden o deben ser aprendidas dentro de un programa integrado, pues no siempre se desarrollan profunda y coherentemente (English, 2016).

El trabajo interdisciplinario es más que la suma de los conocimientos y las prácticas de las áreas que se integran, siendo necesario encontrar puntos de encuentro y de diferencia entre estas (Ortiz-Revilla et al., 2020; Tytler et al., 2019). Se requiere identificar formas en que el aprendizaje en cada disciplina puede distribuirse uniformemente para que los beneficios en un área no reduzcan las ganancias en otras (English, 2016).

Una forma de abordar tal desafío es el desarroIlo de grandes ideas STEM (Clayton et al., 2010; Hurst, 2015; Rennie et al., 2018). Estas se definen como las ideas clave que vinculan numerosos entendimientos disciplinares en conjuntos coherentes que resultan fundamentales para comprender y conocer STEM en una variedad de campos, pudiendo ser idea de contenido (abarcando conceptos, teorías, estrategias y modelos) o ideas de proceso, asociadas a la adquisición y el uso efectivo del conocimiento del contenido (Chalmers et al., 2017; Hurst, 2015). En esta línea, autores como Chalmers et al. (2017) describen el uso de un continuo de grandes ideas STEM de tres posibles formas en la creación de unidades integradas: enfocándose en grandes ideas dentro de una 
disciplina STEM, que tienen aplicación en otras disciplinas STEM; al interdisciplinar grandes ideas de contenido o proceso ubicadas en dos o más disciplinas STEM, reconociendo las diferencias en la forma en que una gran idea se concibe en cada disciplina; y al abarcar grandes ideas conceptuales o grandes ideas que abarcan contenido STEM, que permiten el abordaje interdisciplinario de problemas relevantes.

Considerar las aplicaciones interdisciplinarias de las grandes ideas STEM para promover una compresión profunda de las áreas integradas podría ser una buena forma para mediar un aprendizaje profundo (p. e. Lehrer y Schauble, 2012; Johnson et al., 2016; Silk et al. 2010), pero se requiere determinar cuál es la mejor forma de implementarlas.

\section{Acercando la educación STEM integrada al aula multigrado: marco para el diseño de unidades STEM}

Como hemos argumentado en la sección anterior, se ha destacado la importancia de concebir la integración más allá de la superposición de las áreas que se están integrando, atendiendo las relaciones temporales que se dan dentro de la interdisciplinariedad en STEM (Tytler et al., 2019), reconociendo los puntos de encuentro y diferencia entre las áreas que se integran (Ortiz-Revilla et al., 2020; Tytler et al., 2019), e identificando mecanismos didácticos que permitan desarrollar un aprendizaje profundo y equilibrado en cada una de ellas, respetando sus formas de hacer y pensar propias (Chalmers et al., 2017; English, 2016; Ortiz-Revilla et al., 2020; Tytler et al., 2019). Por tanto, es posible considerar la integración en STEM como una gama de experiencias de aprendizaje que, implicando distintos grados de conexión y temporalidad entre los conocimientos y las prácticas de cada área STEM, buscan promover un aprendizaje profundo y armónico en cada una de ellas.

Consideramos que un enfoque STEM integrado, basado en grandes ideas STEM y la instrucción diferenciada, puede brindar a los niños que se educan en contextos multigrado experiencias significativas en las áreas STEM. Tales experiencias les permitirían comprender en profundidad temáticas relevantes con un carácter global y local, así como desarrollar habilidades del siglo XXI, respetando $y$ atendiendo a sus necesidades individuales y territoriales de desarrollo. La instrucción diferenciada dentro de un plan de estudios integrado puede satisfacer las necesidades de cada estudiante y aumentar sus posibilidades de apropiación de aprendizajes al: (i) ofrecer diversas formas de explorar conceptos básicos y aplicarlos en diversas situaciones, (ii) brindar formas alternativas de dar sentido a las ideas y (iii) generar oportunidades para que los estudiantes puedan expresar en múltiples formas lo que saben (Clayton et al., 2010).

Basados en los aspectos anteriormente señalados, y con el propósito de animar y apoyar a los docentes multigrado a diseñar unidades STEM integradas, proponemos un marco compuesto por tres fases que orienta el diseño de este tipo de unidades, tomando como base grandes ideas STEM y como estrategia la diferenciación, este está resumido en la figura 1. La primera fase, centrada en la estructura de la integración, involucra la selección del tema de la unidad, la identificación y selección de la(s) gran(des) idea(s) STEM asociadas, sus componentes y su proyección en el horizonte de aprendizaje y la evaluación de coherencia y viabilidad de la propuesta en conjunto. La segunda fase, centrada en la planificación general, comprende la reorganización de los aprendizajes de la unidad, su diferenciación, la elaboración de una ruta de aprendizaje y la evaluación de la propuesta en conjunto. La tercera fase, centrada en el diseño de actividades STEM diferenciadas, involucra la elaboración de distintos tipos de actividades STEM para cada nivel en torno a una actividad STEM preliminar, una actividad STEM integrada central, actividades de exploración de grandes ideas STEM, una actividad de consolidación y síntesis, y finalmente la evaluación de la fase. 


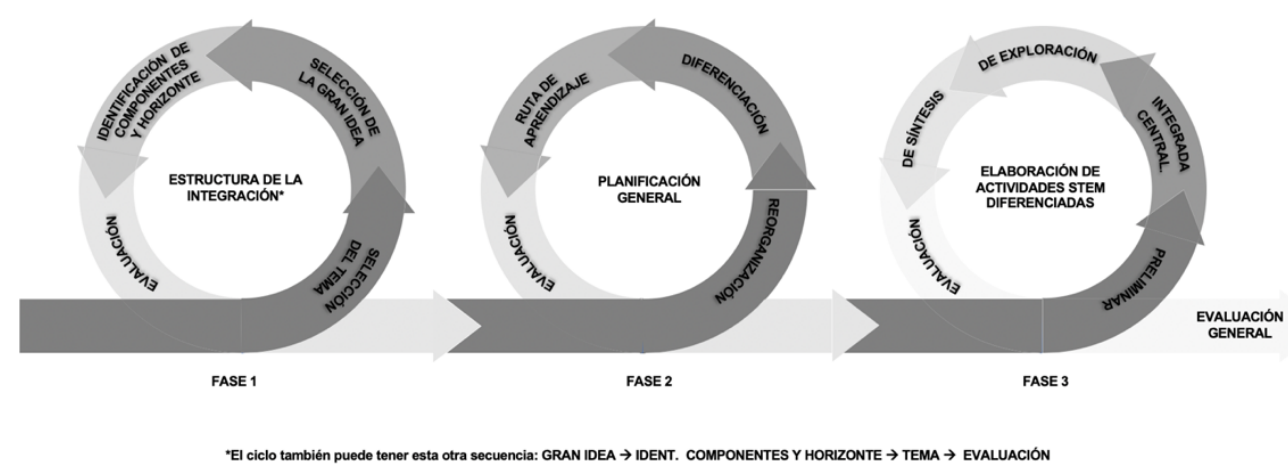

Figura 1. Marco para el diseño de unidades integradas STEM en contextos multigrado.

Fuente: elaboración propia con base en Clayton et al. (2010), Chalmers et al. (2017) y Tomlinson (2014)

\section{Fase 1. Estructura de la integración}

La revisión de documentos curriculares de las disciplinas STEM a integrar permite identificar inicialmente conocimientos y habilidades a desarrollar, visualizar conexiones auténticas, establecer la temática y la(s) gran(des) idea(s) asociada(s), así como los componentes involucrados y su progresión en el currículo (p. e., Chalmers et al,. 2017; Clayton et al., 2010; 2019; 2019; Hurst, 2015; Lehrer y Schauble, 2012). A partir de un tema, se puede(n) determinar la(s) gran(des) idea(s), sus componentes y su proyección en el horizonte de aprendizaje (Chalmers et al., 2017) o determinar el tema a partir de una gran idea junto a sus componentes y horizonte.

Las grandes ideas y los temas deben cumplir ciertos criterios generales para ser abordados en una unidad STEM integrada en contextos multigrado. Se recomienda un número pequeño de grandes ideas para su abordaje profundo (Chalmers et al., 2017), el análisis de aspectos propios del territorio, así como la graduación de los saberes a desarrollar y su pertinencia. Cabe preguntarse, por ejemplo: ¿la(s) gran(des) idea(s) seleccionada(s) es(son) abordada(s) coherentemente en distintos grados de profundidad en todos los niveles?, ¿qué componentes involucrados en esta(s) idea(s) pueden ser abordados en cada nivel?, ¿el tema es motivador y relevante para la vida de todos los estudiantes?, ¿permite un abordaje interdisciplinario y el desarrollo de al menos una gran idea STEM? (Chalmers et al., 2017; Clayton et al., 2010).

Posteriormente, se evalúa la coherencia y viabilidad de la propuesta antes de pasar a la siguiente fase, siendo fundamental incluir la revisión de las progresiones de aprendizaje de los temas propios de cada disciplina que no serán parte de la unidad integrada, para que no se vean afectados en su desarrollo dentro de cada una de estas (Clayton et al., 2010).

Así, como ejemplo concreto, es posible abordar la crisis hídrica vinculada al cambio climático, tema de alta pertinencia para una comunidad educativa de una isla de la región de Los Lagos, en el sur austral de Chile. Esta zona geográfica presenta problemas de déficit hídrico, siendo necesaria la concientización y la reflexión para promover la capacidad de agencia frente al problema.

Con base en esta elección, se revisan programas de estudio (Mineduc, 2018), Orientaciones Pedagógicas (Mineduc, 2017a y 2017b) y documentos sobre cambio climático (p. e. Ministerio del Medio Ambiente, 2017), entre otros, visualizando la crisis hídrica como una gran idea que abarca contenido STEM. Seguidamente, se identifican los componentes involucrados en esta idea, recogidos en los diferentes ejes o temas en los que se organiza el currículo. En el currículo de ciencias naturales esta idea involucra el estudio del agua y su importancia para la vida, el tiempo atmosférico, el 
clima y sus efectos en el medio ambiente y el estudio de las plantas y el suelo. En matemáticas, la localización, la medición de diversas magnitudes temporales y espaciales, y la representación y el análisis de la información a través de los datos. A partir de este análisis, se seleccionan los componentes clave que permiten la comprensión de esta gran idea y se evalúa la coherencia y viabilidad de la propuesta.

\section{Fase 2. Planificación general}

Secuenciar lógicamente los aprendizajes abordados implica una reorganización de los aprendizajes de cada área STEM a abordar para el desarrollo de la temática y de la(s) gran(des) idea(s), con el fin de visualizar cómo se desarrollarán los subtemas en la secuencia (Clayton et al., 2010). Para ello, resulta conveniente reorganizarlos en función de los cuatro tipos de actividades STEM: una actividad STEM preliminar que introduce a los alumnos al estudio de la problemática o el desafío, una actividad STEM integrada central en la que este se aborda, actividades de exploración de grandes ideas STEM que buscan establecer un vínculo cognitivo entre las grandes ideas involucradas y sus representaciones disciplinares más específicas, y una actividad de consolidación y síntesis en la que se conectan ideas específicas para dar respuesta al desafío o problema (Clayton et al., 2010; Chalmers et al., 2017).

Posteriormente, es necesario establecer una diferenciación de los aprendizajes involucrados en cada nivel, considerando la profundidad en que se debe(n) abordar la(s) gran(des) idea(s) STEM y sus componentes, así como su articulación para promover la circulación de saberes. Resulta conveniente bosquejar una ruta de aprendizaje para visualizar el desarrollo de la unidad en cada nivel, formulando preguntas clave que dividan el conocimiento que involucra el desafío o problema en partes más pequeñas para ser abordadas gradualmente y establecer desempeños (Clayton et al., 2010).
La ruta de aprendizaje facilita una mirada horizontal y vertical del tema y de los aprendizajes que se espera desarrollar. La mirada horizontal permite visualizar la forma en que las grandes ideas y sus componentes se desarrollan con distinta profundidad a través de los grados, evaluar su coherencia y pertinencia, identificar la contribución de cada nivel al desafío/pregunta/problema, asignar tareas, y definir espacios de trabajo individual, colaborativo y de circulación de saberes. La mirada vertical permite determinar la forma en que se desarrollará temporalmente la integración, visualizando el mecanismo con el que las ideas disciplinarias involucradas se abordarán para desarrollar un aprendizaje profundo y equilibrado en cada una de las áreas, y para establecer conexiones entre las grandes ideas involucradas (Chalmers et al., 2017; English, 2016; Tytler et al., 2019). Finalmente, se evalúa la coherencia y viabilidad de la propuesta.

Retomando el ejemplo anterior, se reorganizan los aprendizajes a abordar durante la secuencia en función de los cuatro tipos de actividades STEM, estableciendo una secuencia lógica para su desarrollo y los desempeños esperados. Seguidamente, se establece una diferenciación de los aprendizajes que se abordarán en los diferentes grados en la unidad y se esboza una ruta de aprendizaje (ver tabla 1).

\section{Fase 3. Elaboración de actividades STEM diferenciadas}

A partir de la ruta de aprendizaje, se elabora una actividad STEM preliminar, considerando una actividad troncal para todos los niveles que introduzca a los alumnos en el estudio de la problemática o el desafío que se abordará, permita la activación de conocimientos previos y el alcance de las ideas base para el desarrollo de la unidad. En el ejemplo señalado una posible actividad contempla un recorrido por determinados sectores de la isla, donde los estudiantes exploren y analicen mediante recolección, registro e interpretación de datos, según cada nivel, la importancia del agua para la vida y la 
Tabla 1. Extracto de la ruta de aprendizaje

\begin{tabular}{|c|c|c|c|c|c|c|}
\hline \multirow{3}{*}{$\begin{array}{l}\text { Pregunta guía y } \\
\text { temas a abordar }\end{array}$} & \multirow{3}{*}{ Idea clave } & \multicolumn{5}{|c|}{ Diferenciación } \\
\hline & & \multicolumn{5}{|c|}{ Desempeños esperados por nivel } \\
\hline & & $1^{\circ}$ & $2^{\circ}$ & $3^{\circ}$ & $4^{\circ}$ & $5^{\circ}$ y $6^{\circ}$ \\
\hline $\begin{array}{l}\text { ¿Cómo podemos } \\
\text { ayudar a nuestra } \\
\text { comunidad a } \\
\text { hacer un buen } \\
\text { uso del agua } \\
\text { dulce? } \\
\text { Temas } \\
\text {-Estados del agua } \\
\text {-Fuentes de } \\
\text { agua dulce y su } \\
\text { localización. }\end{array}$ & $\begin{array}{l}\text { Idea 1. El agua se } \\
\text { puede encontrar en } \\
\text { distintos estados. } \\
\text { Idea 2. El agua } \\
\text { dulce proviene de } \\
\text { distintas fuentes } \\
\text { en la Tierra y se } \\
\text { distribuye en } \\
\text { determinados } \\
\text { puntos del territorio } \\
\text { que se pueden } \\
\text { localizar. } \\
\text { Idea } 3 . \text { La posición } \\
\text { de un objeto es } \\
\text { aquella información } \\
\text { que permite } \\
\text { localizarlo en el } \\
\text { espacio en un } \\
\text { instante de tiempo } \\
\text { determinado. }\end{array}$ & $\begin{array}{l}\text { Observar, por medio } \\
\text { de la exploración, } \\
\text { algunas características } \\
\text { del agua (por } \\
\text { ejemplo, escurrir } \\
\text { o adaptarse a la } \\
\text { forma del recipiente, } \\
\text { disolver algunos } \\
\text { sólidos, evaporarse } \\
\text { y congelarse con } \\
\text { los cambios de } \\
\text { temperatura). } \\
\text { Describir la posición } \\
\text { de fuentes de agua } \\
\text { con respecto a sí } \\
\text { mismos y a otros } \\
\text { objetos en la isla } \\
\text { utilizando un lenguaje } \\
\text { común (arriba, abajo, } \\
\text { izquierda y derecha, } \\
\text { etc.) }\end{array}$ & $\begin{array}{l}\text { Observar y } \\
\text { describir, por } \\
\text { medio de la } \\
\text { experimentación, } \\
\text { algunas } \\
\text { características del } \\
\text { agua, identificando } \\
\text { y comparando } \\
\text { los estados: } \\
\text { sólido, líquido y } \\
\text { gaseoso. Describir } \\
\text { el ciclo del agua } \\
\text { en la naturaleza, } \\
\text { relacionándolo } \\
\text { con diferentes } \\
\text { referentes } \\
\text { geográficos de la } \\
\text { isla y su posición, } \\
\text { por medio de la } \\
\text { representación a } \\
\text { través de material } \\
\text { concreto y dibujos. }\end{array}$ & $\begin{array}{l}\text { Describir la } \\
\text { localización } \\
\text { de fuentes de } \\
\text { agua dulce } \\
\text { en la isla a } \\
\text { través de un } \\
\text { mapa simple o } \\
\text { cuadrícula. }\end{array}$ & $\begin{array}{l}\text { Describir la } \\
\text { localización de } \\
\text { fuentes de agua } \\
\text { dulce y salada } \\
\text { en la isla a través } \\
\text { de un mapa } \\
\text { simple con } \\
\text { coordenadas } \\
\text { informales } \\
\text { (por ejemplo, } \\
\text { con letras y } \\
\text { números), y la } \\
\text { localización } \\
\text { relativa en } \\
\text { relación con } \\
\text { otros objetos. }\end{array}$ & $\begin{array}{l}\text { Identificar y } \\
\text { dibujar puntos } \\
\text { en el primer } \\
\text { cuadrante del } \\
\text { plano cartesiano } \\
\text { utilizando } \\
\text { coordenadas en } \\
\text { números naturales } \\
\text { que representen } \\
\text { fuentes de agua } \\
\text { dulce y salada en } \\
\text { la isla. } \\
\text { Reconocer la } \\
\text { escasez relativa } \\
\text { de agua dulce, } \\
\text { en comparación } \\
\text { con las fuentes de } \\
\text { agua en la Tierra, a } \\
\text { través del estudio } \\
\text { de sus volúmenes. }\end{array}$ \\
\hline
\end{tabular}

Fuente: Elaboración propia con base en Mineduc (2018).

preservación de distintos tipos de hábitats, especies y su comunidad, o evalúen cómo se ha visto afectada en el tiempo la disponibilidad de agua.

Posteriormente, se debe definir una actividad STEM integrada central integrada para abordar el desafío o problema, que se completa al final de la secuencia cuando se aplican grandes ideas STEM que son relevantes en más de una disciplina. Esta actividad debe tener sentido y permitir que todos los alumnos contribuyan coherentemente a la solución en su respectivo nivel. En el caso de nuestro ejemplo, corresponde a la pregunta: ¿cómo podemos ayudar a nuestra comunidad a hacer un buen uso del agua dulce? Para darle respuesta, en una primera instancia, es necesario que los alumnos comprendan que el agua dulce se encuentra en diferentes estados, proviene de diferentes fuentes y se distribuye en determinados puntos localizables de la isla para optimizar su uso. Los estudiantes pueden hacer un estudio geográfico de la isla, aportando en diferentes formas a la elaboración de un prototipo de modelo que permita observar la distribución del agua dulce en el territorio, pudiendo complementarse con mapas virtuales y el conocimiento de la comunidad.

También es necesario considerar actividades de exploración de grandes ideas STEM, orientadas a establecer un vínculo cognitivo entre las grandes ideas involucradas en el desafío o problema y sus representaciones más específicas en cada disciplina. Es importante considerar actividades que permitan explorar cómo cada idea vinculada al desafío o problema funciona dentro de su disciplina. En el caso del ejemplo utilizado, estas buscan establecer un vínculo cognitivo entre cinco construcciones entrelazadas (medida, clima, agua, suelo y plantas) para asociar la falta de agua dulce, acrecentada en zonas de escasa vegetación, como consecuencia de una variación climática. Así, por ejemplo, dependiendo de cada nivel, los alumnos 
investigan características o efectos de los cambios del tiempo atmosférico y sus consecuencias para los seres vivos y el ambiente, estudian la vegetación presente en la isla o la conformación del suelo, activando el sentido de la medida y cómo funciona en diferentes contextos.

Finalmente, se debe incluir una actividad de consolidación y síntesis, que ofrezca un contexto para que los alumnos conecten las ideas específicas desarrolladas en un conocimiento interdisciplinario que responda al problema planteado en diferentes formas. Así, por ejemplo, los alumnos pueden elaborar material de difusión de su hallazgos y aprendizajes y realizar una exposición a la comunidad sobre diversas acciones cotidianas para el cuidado del agua o para mejorar la disponibilidad de agua dulce, como aquellas asociadas al cuidado del suelo y las plantas en determinados puntos del territorio insular, según el nivel en que se encuentren.

\section{Reflexiones finales}

En este artículo hemos evidenciado algunos de los principales desafíos que enfrenta la enseñanza en contextos multigrado, así como el potencial de la educación STEM integrada para abordarlos. A la fecha no existe claridad sobre cuál es la mejor forma de apoyar a los docentes multigrado para superar las dificultades que involucra adaptar el currículo para una instrucción diferenciada (p. e. Abós Olivares y Boix, 2017; Boix y Bustos, 2014; Ribadeneira, 2020), ni cómo responder al abordaje de problemáticas locales y globales con un carácter interdisciplinar, atendiendo la heterogeneidad del aula.

Argumentamos que una forma de hacer frente a estos desafíos es alentar y apoyar a los maestros a diseñar unidades STEM integradas. El aprendizaje activo e interdisciplinario que se genera bajo el enfoque STEM integrado ofrece experiencias de aprendizaje relevantes y auténticas que permiten a los estudiantes adquirir los conocimientos y las habilidades necesarias para participar activamente en problemáticas relevantes y desafíos del futuro, atendiendo a sus diferencias individuales.

A pesar de que la enseñanza interdisciplinaria es reconocida como una estrategia efectiva, los maestros tienen dificultades para establecer conexiones interdisciplinarias para la instrucción en el aula (Brand y Triplett, 2012). En este contexto, el marco presentado en este estudio ofrece un insumo para ilustrar cómo brindar oportunidades de aprendizaje integrado con base en proyectos o problemas que aborden temáticas pertinentes y relevantes que potencien el desarrollo de habilidades del siglo XXI. A su vez, permite acercar la educación STEM a entornos en los que normalmente no se tiene acceso. Las proyecciones de este estudio contemplan evaluar los efectos de la implementación de este modelo en el aula, así como las percepciones de los docentes multigrado sobre su viabilidad.

\section{Agradecimientos}

Esta investigación es financiada por el proyecto $\mathrm{PI}$ FID UACh Mineduc 023-2019.

\section{Referencias}

Abós Olivares, P., Boix, R. (2017). Evaluación de los aprendizajes en escuelas rurales multigrado. Aula abierta, 45(1), 41-48. https://doi.org/10.17811/ rifie.45.2017.41-48

Abós Olivares, P., Bustos-Jiménez, A. (2015). Teaching strategies and space organisation in multigrade classrooms. Sisyphus Journal of Education, 3(2), 5877. https://doi.org/10.25749/sis.7886

Bennet, C., Ruchti, W. (2014). Bridging STEM with mathematical practices. Journal of STEM Teacher Education, 49 (1),17-28. https://doi.org/10.30707/ jste49.1bennett

Boix, R., Bustos, A. (2014). La enseñanza en las aulas multigrado: una aproximación a las actividades escolares y los recursos didácticos desde la perspectiva del profesorado. Revista Iberoamericana de Evaluación Educativa, 7(3), 29-43 
Blackley, S., Howell, J. (2015). A STEM narrative: 15 years in the making. Australian Journal of Teacher Education, 40(7), 102-112. https://doi. org/10.14221/ajte.2015v40n7.8

Bosch, H., Di Blasi, M., Pelem, M., Bergero, M., Carvajal, L., Geromini, N. (2011). Nuevo paradigma pedagógico para enseñanza de ciencias y matemática, Avances en Ciencias e Ingeniería, (2)3, 131-140

Brand, B. R., Triplett, C. F. (2012). Interdisciplinary curriculum: An abandoned concept? Teachers and Teaching: Theory and Practice, 18(3), 381-393. https://doi.org/10.1080/13540602.2012.629847

Breiner, J. M., Harkness, S. S., Johnson, C. C., Koehler, C. M. (2012). What is STEM? A discussion about conceptions of STEM in education and partnerships. School Science and Mathematics, 112(1), 3-11. https://doi.org/10.1111/j.1949-8594.2011.00109.x

Bustos, A. (2014). La didáctica multigrado y las aulas rurales: perspectivas y datos para su análisis. Innovación educativa, 24, 119-131. https://doi. org/10.15304/ie.24.1994

Castro, A., Iturbe, C., Jiménez, R., Silva., R. (2020). ¿Educación STEM o en humanidades? Una reflexión en torno a la formación integral del ciudadano del siglo XXI. Utopía y Praxis Latinoamericana, 25(9), 197-208. https://doi.org/10.5281/zenodo.4110904

Chalmers, C., Carter, M. L., Cooper, T., Nason, R. (2017). Implementing "big ideas" to advance the teaching and learning of science, technology, engineering, and mathematics (STEM). International Journal of Science and Mathematics Education, 15(1), 25-43. https://doi.org/10.1007/s10763-017-9799-1

Clayton, M., Hagan, J., Ho, P. S., Hudis, P. M. (2010). Designing Multidisciplinary Integrated Curriculum Units. ConnectED: The California Center for College and Career

English, L. D. (2016). STEM education K-12: Perspectives on integration. International Journal of STEM Education, 3, e3 https://doi.org/10.1186/ s40594-016-0036-1

English, L. D., King, D. T. (2015). STEM learning through engineering design: Fourth-grade students' investigations in aerospace. International Journal of
STEM Education, 2, e14. https://doi.org/10.1186/ s40594-015-0027-7

English, L. D., King, D. (2019). STEM integration in sixth grade: Desligning and constructing paper bridges. International Journal of Science and Mathematics Education, 17(5), 863-884. https://doi. org/10.1007/s10763-018-9912-0

Gresnigt, R., Taconis, R., van Keulen, H., Gravemeijer, K., Baartman, L. (2014). Promoting science and technology in primary education: A review of integrated curricula. Studies in Science Education, 50(1), 47-84. https://doi.org/10.1080/03057267.2013.877694

Guzey, S. S., Moore, T. J., Harwell, M. (2016). Building up STEM: An analysis of teacher-developed engineering design-based STEM integration curricular materials. Journal of Pre-College Engineering Education Research (J-PEER), 6(1), 11-29. https://doi. org/10.7771/2157-9288.1129

Honey, M. A., Pearson, G., Schweingruber, H. (2014). STEM integration in K-12 education: Status, prospects, and an agenda for research. Washington, DC: The National Academies Press. https://doi. org/10.17226/18612

Hurst, C. (2015). Thinking big about mathematics, science, and technology: Effective teaching STEMs from big ideas. International Journal of Innovation in Science and Mathematics Education, 23(3), 11-21

Hyry-Beihammer, E. K., Hascher, T. (2015). Multigrade teaching in primary education as a promising pedagogy for teacher education in Austria and Finland. En C. Craig \& L. Orland-Barak (Eds.) International Teacher Education: Promising Pedagogies (Part C) (Advances in Research on Teaching, Vol. 22C) (pp. 89-113). Bingley: Emerald Group Publishing Limited. https://doi.org/10.1108/ $\underline{\text { S1479-368720150000022005 }}$

Johnson, C. C., Peters-Burton, E. E., Moore, T. J. (Eds.). (2016). STEM Road Map: A Framework for Integrated STEM Education. Routledge

Koul, R., Fraser, B., Nastitia, H. (2018). Transdisciplinary instruction: Implementing and evaluating a primary-school STEM teaching model. International Journal of Innovation in Science and Mathematics Education, 26(8), 17-19 
Lehrer, R., Schauble, L. (2012). Seeding evolutionary thinking by engaging children in modeling its foundations. Science Education, 96(4), 701-724. https:// doi.org/10.1002/sce.20475

McDonald, C. V. (2016). STEM education: A review of the contribution of the disciplines of science, technology, engineering and mathematics. Science Education International, 27(4), 530-569

Ministerio de Educación. (2017a). Orientaciones pedagógicas para el aula multigrado Matemáticas. https://rural.mineduc.cl/wp-content/uploads/sites/22/2018/05/AULA_MULTIGRADO-Matematica.pdf

Ministerio de Educación. (2017b). Orientaciones pedagógicas para el aula multigrado Ciencias Naturales. https://rural.mineduc.cl/wp-content/uploads/ sites/22/2018/05/AULA MULTIGRADO Ciencias-Naturales.pdf

Ministerio de Educación. (2018). Bases curriculares. Primero a sexto básico. https://bibliotecadigital. mineduc.cl/bitstream/handle/20.500.12365/2342/ mono-1003.pdf

Ministerio del Medio Ambiente. (2017). Guía de apoyo docente en cambio climático. https://mma.gob.cl/ wp-content/uploads/2018/08/Guia-de-apoyo-docente-en-Cambio-Climatico.pdf

Moore, T., Johnson C., Peters-Burton, E.,Guzey, S. (2015). The need for a STEM road map. En C. C. Johnson, E. E. Peters-Burton, \& T. J. Moore (Eds.), STEM Road Map: A Framework for Integrated STEM Education (pp. 3-12). Routledge. https://doi. org/10.4324/9781315753157-1

Nadelson, L. S., Seifert, A. L. (2017). Integrated STEM defined: Contexts, challenges, and the future. The Journal of Educational Research, 110(3), 221-223. https://doi.org/10.1080/00220671.2017.1289775

Nadelson, L. S., Seifert, A. L. (2019). Teaching and learning integrated STEM: Using andragogy to foster an entrepreneurial mindset in the age of synthesis. En A. Sahin \& M. J. Mohr-Schroeder (Eds.), STEM Education 2.0 (pp. 53-71). Brill. https://doi. org/10.1163/9789004405400_004

Nesmith, S. M., Cooper, S. (2019). Engineering process as a focus: STEM professional development with elementary STEM-focused professional development schools. School Science and Mathematics, 119(8), 487-498. https://doi.org/10.1111/ssm.12376

$\mathrm{Ng}$, O. L., Chan, T. (2019). Learning as making: Using 3D computer-aided design to enhance the learning of shape and space in STEM-integrated ways. British Journal of Educational Technology, 50(1), 294308. https://doi.org/10.1111/bjet.12643

Ortiz-Revilla, J., Adúriz-Bravo, A., Greca, I. M. (2020). A Framework for epistemological discussion on integrated STEM education. Science \& Education, 29(4), 857-880. https://doi.org/10.1007/ s11191-020-00131-9

Peirano, C., Estévez, S. P. y Astorga, M. I. (2015). Educación rural: oportunidades para la innovación. Cuadernos de Investigación Educativa, 6(1), 53-70. https://doi.org/10.18861/cied.2015.6.1.7

Rennie, L., Venville, G., Wallace, J. (2018). Making STEM curriculum useful, relevant, and motivating for students. En R. Jorgensen, \& K. Larkin (Eds.), STEM Education in the Junior Secondary (pp. 91-109). Singapur: Springer. https://doi. org/10.1007/978-981-10-5448-8 6

Ribadeneira, F. M. (2020). Estrategias didácticas en el proceso educativo de la zona rural. Conrado, 16(72), 242-247

Shealer, R., Shealer, M. (2014). Making it real: A cooperative, multigrade, $3 \mathrm{D}$ design project. Technology and Engineering Teacher, 74(2), 8-11

Shareefa, M. (2021). Using differentiated instruction in multigrade classes: A case of a small school. Asia Pacific Journal of Education, 41(1), 167-181. https://doi.org/10.1080/02188791.2020.1749559

Sias, C. M., Nadelson, L. S., Juth, S. M., Seifert, A. L. (2017). The best laid plans: Educational innovation in elementary teacher generated integrated STEM lesson plans. The Journal of Educational Research, 110(3), 227-238. https://doi.org/10.1080/0022067 1.2016 .1253539

Silk, E. M., Higashi, R., Shoop, R., Schunn, C. D. (2010). Designing technology activities that teach mathematics. The Technology Teacher, 69(4), 21-27

Smit, R., Humpert, W. (2012). Differentiated instruction in small schools. Teaching and 
Teacher Education, 28(8), 1152-1162. https://doi. org/10.1016/j.tate.2012.07.003

Struyf, A., De Loof, H., Boeve-de Pauw, J., Van Petegem, P. (2019). Students' engagement in different STEM learning environments: Integrated STEM education as promising practice? International Journal of Science Education, 41(10), 1387-1407. https://doi. org/10.1080/09500693.2019.1607983

Taole, M. J. (2017). Identifying the professional knowledge base for multi-grade teaching. Gender and Behaviour, 15(4), 10419-10434

Taole, M. J. (2020). Diversity and inclusion in rural South African multigrade classrooms. International Journal of Inclusive Education, 24(12), 12681284. https://doi.org/10.1080/13603116.2018.1 $\underline{520310}$

Tomlinson, C. A. (2014). The Differentiated Classroom: Responding to the Needs of All Learners. ASCD
Tati, T., Firman, H., Riandi, R. (2017). The effect of STEM learning through the project of designing boat model toward student STEM literacy. Journal of Physics: Conference Series, 895, e012157. https://doi. org/10.1088/1742-6596/895/1/012157

Tytler, R., Prain, V., Hobbs, L. (2019). Rethinking disciplinary links in interdisciplinary STEM learning: A temporal model. Research in Science Education, 2019, 1-19. https://doi.org/10.1007/s11165-019-09872-2

UNESCO. (2015). Practical Tips for Multigrade Teaching Classes. París: United Nations Educational, Scientific and Cultural Organization. https://www.eenet. org.uk/resources/docs/ilfe/generic/Sp4.pdf

Vera-Bachman, D., Salvo, S. (2016). Perfiles de escuelas rurales exitosas: una propuesta de la psicología educacional ante el cierre de escuelas. Universitas Psychologica, 15(2), 25-38. https://doi.org/10.11144/ laveriana.upsy15-2.perep 